\title{
Article \\ Development and Fabrication of an Innovative Smart Tool to Monitor the Impact Carving Process on Brittle Stones and Marble
}

\author{
Nectarios Vidakis ${ }^{1}$, Markos Petousis ${ }^{1, *(\mathbb{D})}$, Athena Maniadi ${ }^{2}(\mathbb{D})$ and Emmanuel Arapis ${ }^{1}$ \\ 1 Mechanical Engineering Department, Hellenic Mediterranean University, 71410 Heraklion, Greece; \\ vidakis@hmu.gr (N.V.); arapis.man@hotmail.com (E.A.) \\ 2 Department of Materials Science and Technology, University of Crete, 70013 Heraklion, Greece; \\ maniadi@materials.uoc.gr \\ * Correspondence: markospetousis@hmu.gr; Tel.: +30-2810379227
}

Citation: Vidakis, N.; Petousis, M.; Maniadi, A.; Arapis, E. Development and Fabrication of an Innovative Smart Tool to Monitor the Impact Carving Process on Brittle Stones and Marble. Electronics 2022, 11, 12. https://doi.org/10.3390/ electronics11010012

Academic Editor: Chiman Kwan

Received: 14 November 2021

Accepted: 21 December 2021

Published: 22 December 2021

Publisher's Note: MDPI stays neutral with regard to jurisdictional claims in published maps and institutional affiliations.

Copyright: (C) 2021 by the authors. Licensee MDPI, Basel, Switzerland. This article is an open access article distributed under the terms and conditions of the Creative Commons Attribution (CC BY) license (https:// creativecommons.org/licenses/by/ $4.0 /$ )

\begin{abstract}
The art of sculpting is related to the processing of brittle materials, such as granite, marble, and stone, and is implemented using percussive hand tools or rotational roughing tools. The outcome of percussion carving is still directly related to the technique, experience, and capacity of the sculptor. Any attempt to automate the art of sculpturing is exhausted in the subtraction method of brittle materials using a rotating tool. In the process of percussion carving, there is no equivalent expertise In this work, we present the design, manufacturing (3D printing and CNC machining), and use of a smart, percussion carving tool, either manually by the hand of a sculptor, adjusted in a percussive pneumatic hammer, or guided by a digitally driven machine. The scope is to measure and record the technological variables and sizes that describe and document the carving process through the sensors and electronic devices that the smart tool incorporates, the development and programming of which was implemented for the purposes of this work. The smart carving tool was meticulously tested in various carving stones and stressing scenarios to test the functionality and efficacy of the tool. All the tests were successfully implemented according to the specifications set.
\end{abstract}

Keywords: sculpture; arduino; marble; design; carving tool; prototyping; chisel

\section{Introduction}

The art of percussion carving has remained unchanged over the past decades. The sculptor, with simple hand tools (chisel and hammer), moves with the whole body the chisel in various gradients and orientations, while exercising the flat appreciation (empirical) of optimal pressure on the tool to achieve the artistic result with the selected stone material. Stonework is a longstanding tradition encompassing a multitude of techniques for stereotomy, stone dressing, and finishing [1,2].

While almost all manufacturing processes are mathematically modeled and fully supported by automated numerical control machines, the art of percussion carving remains empirical and hand driven. As skilled craftsmen diminish in number, digital fabrication methods demonstrate the potential to form new links between computational tools, robotic systems, and craftsmanship [3,4]. Current digital carving technology is widely applied in machine processing and die making and produces preferable economic benefits. However, its functions are limited to digital art-carving aspects [5].

Stone, as a material, is a complex and fragile substrate to handle, with irregular geometry and uneven, coarse texture. Therefore, applying an uncontrolled force may result in unpredictable outcomes [6]. The core of stonework lies in the sensitivity applied by the stone carvers when using carving tools of any kind. This sensitivity allows them to respond differently to the unpredictability of each stone [7]. This form of unpredictability is manually dealt with through continuous decision-making and motion adjustments. The sculpturing process at the moment is either exercised by hand or automated with computer 
numeric control machines using rotating tools. In the first case, the result depends on the talent and the capabilities of the sculptor which cannot be at the moment logged and quantified. In the second case, the automated processes due to the rotational tools used, provide inferior results in the sculptures which cannot be compared to authentic hand-made ones.

This project aimed to create a novel medium, a smart percussion carving tool capable of processing brittle materials used in sculpturing (marble, granite, limestone, soapstone, etc.) that can map the entire carving process, either in the hands of an experienced sculptor or even in a fully automated sculpting machine. The smart tool was manufactured mostly by stainless steel, which incorporates sensors and electronic systems on the inside of the main part to save and export measurements, such as the tool's load and forces upon it, carving frequency, angles in three axes, angle speeds in three axes, linear velocities in all three axes, and temperatures wirelessly via Bluetooth or via micro-SD card and USB cable. The current market for sculpting tools does not have a smart tool for such capabilities. Additionally, literature in the field is very limited. Following the previous statements, a patent was filed for both the smart carving tool (child patent) and for the mechatronic sculptor machine which will be filled soon (parent patent).

The smart percussion carving tool was designed and manufactured to act both as a manual hand tool or attached in a pneumatic hammer or as a complete smart tool system attached to a digitally guided carving machine, which can design and manufacture its front chisel part removed and replaced by any type of chisel available. In addition to the ability to replace the front chisel part, the tool can be used in all carving procedures. It also incorporates the ability to replace the strain gauges' part and replace the back piece that is being hit by the hammer. This gives the end user the choice and the freedom to change how the tool responds to each stone and each carving procedure and adapts the tool to current needs while mapping the entire carving process. In the final steps of this work, the smart carving tool was tested in real carving scenarios and benchmark testing and was found to be fully functional and ready to interpret the art of percussion carving.

\section{Materials and Methods}

\subsection{Designing Methodology: The Tool's Requirements}

As shown in Figure 1, the design methodology is based on the basic requirements that a tool should incorporate. This smart carving tool should be held with ease, by an average size, female, or male hand, without being very different from a conventional sculpturing chisel. The smart carving tool should be capable of operating as a traditional sculpting chisel, accepting heavy loads with a hammer or pneumatic hammer at medium frequency, and being able to have interchangeable points and parts. According to the previous statement, the smart carving tool should have proper dimensions to be single-handheld, robust to withstand heavy loads, and have replaceable parts to meet the sculptors' needs.

Regarding the electronic requirements, autonomy, and connectivity, a smart tool should be able to measure and store all the data of the tool's orientation, speed, acceleration, temperature, load, and frequency in real-time, while being handheld without any wires. Thus, the smart tool should incorporate all the required electronic sensors and processors, memory, battery module, and connectivity modules to measure, save, and transfer these sculpturing parameters via wireless data transfer to the nearest station (PC/laptop/tablet).

\subsection{Mechanical Parts Designing and Manufacturing Methodology}

Following the specifications set, a typical top-down and bottom-up methodology was followed for the design process. The tool design and the design of its manufacturing process were performed using computer-aided design (CAD) and computer-aided manufacturing (CAM) platforms, respectively. The parts of the smart tool were designed to conform to the required specifications and to have a suitable geometry to host the device's electrical and electronic equipment. 3D geometric models were initially developed to evaluate the design on a computer. As expected, several editions were produced to overcome the issues 
determined during the process, with the aim of achieving a design fully compatible with the specifications. The limited space required to maintain an ergonomic design for the user's hand, for fitting the electrical and electronic devices in the smart tool, was also a design challenge in the process. Other parameters were also considered, such as the required strength and the ability to manufacture smart tool parts with stainless steel in a machine shop. All the versions designed were then 3D printed to evaluate the design, the required space for the electric and electronic devices, and the feasibility of the smart tool parts to be assembled and disassembled. Physical prototypes were 3D printed on a Dimension Elite 3D printer (Stratasys, Ltd., Edina, MN, USA) with Stratasys acrylonitrile butadiene styrene (ABS) plus material.

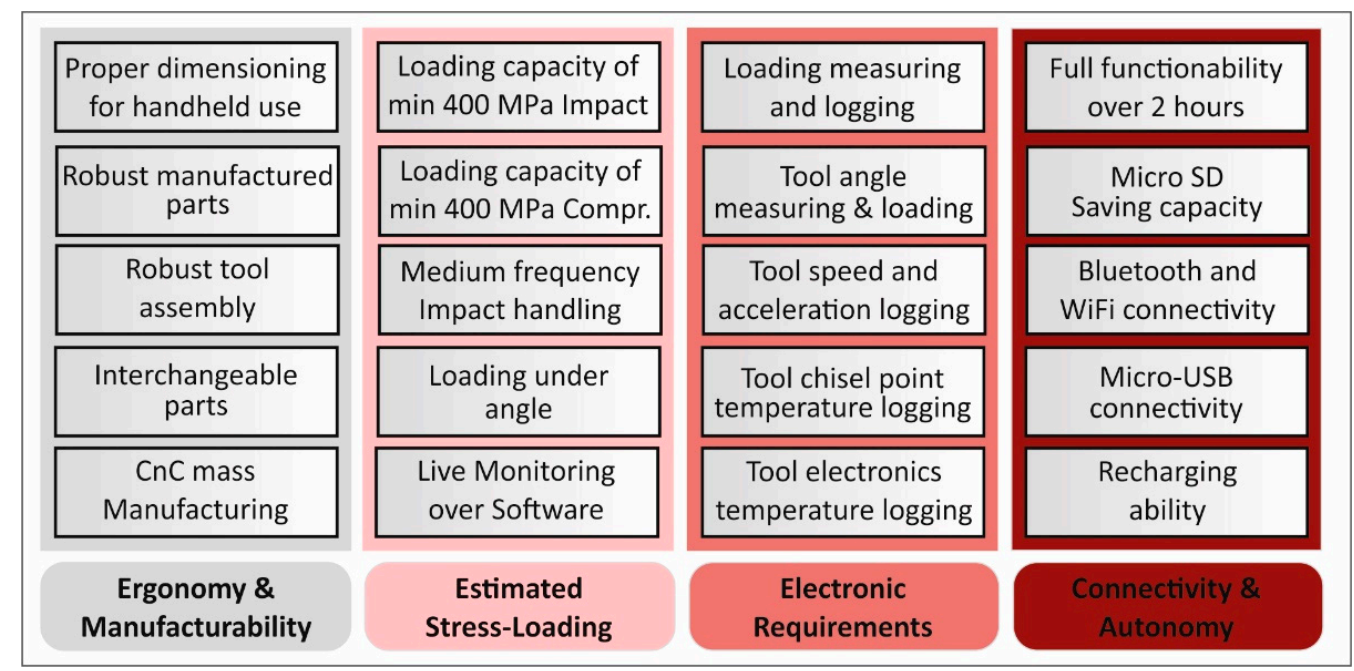

Figure 1. Smart percussion carving tool, initial designing, and electronic requirements.

After several repetitions of the process and the completion of the 3D design of the smart carving tool, the optimized design of the smart tool was then manufactured. Computeraided manufacturing (CAM) software platform supported the process, setting the cutting parameters, cutting tools, and extracting the G-Code to the laboratory's CNC 4 axis milling machines. CNC parts were manufactured on a Haas VF2 4 axis milling machine center (Haas Ltd., Oxnard, CA, USA).

Most parts of the smart tool require both conventional processing and CNC machining. The overall time required for the complete manufacturing of the tool part was $12 \mathrm{~h}$ of conventional lathe machining and $30 \mathrm{~h}$ of $\mathrm{CNC}$ milling. After the completion of the parts, every metallic part was checked thoroughly for manufacturing faults and polished to obtain a good product finish.

The selected material for manufacturing the smart percussion carving tool was 304 stainless steel (Fe, C < 0.08\%, Cr 17.5-20\%, Ni 8-11\%, Mn <2\%, Si < 1\%, P < 0.045\%, S < 0.03\%), while the electronic enclosure base part was manufactured with Ertacetal, and three (3) chisel parts were manufactured with 1.6582 (CrNiMo 34) steel. The selected steel materials have excellent machinability, very good corrosion resistance, and high mechanical strength, which is needed for this type of percussion tool.

\subsection{Smart Tool's Electronical Structuring Methodology \\ 2.3.1. Tool's Electronical System Design}

The technological parameters and variables describing the percussion carving process in stone and brittle materials remain unknown. Percussive carving is a process based on the technique, experience, and skill of each sculptor. Therefore, all parameters defying the results are achieved empirically by the sculptor. The basic parameters that must be identified are the exerted force, linear acceleration, orientation, and temperature. 
To address these technological quantities that surround the percussion carving process, the tool must incorporate the appropriate electronic devices and sensors to be obtained, analyzed, and recorded in real-time as an autonomous device. Based on the assumption above, the sensors that had to be integrated into the tool were a mechanical load unit, an inertial measurement unit (IMU), and a temperature measurement unit.

The mechanical load unit measures the forces exerted on the tool. The IMU incorporates sensors that can measure the angular velocity, angular acceleration, and magnetic field intensity in three axes and the temperature. From these IMU measurements, the linear acceleration can be calculated in three axes and the orientation in space at the current time. The smart tool features two (2) temperature sensors. One is installed near the chisel's tip, to monitor the impact head's temperature. This temperature is crucial cause it is directly connected to the chisel's wear and fatigue due to the impact stress and dynamic loading during its use. This data are important if the tool is to be attached to a CNC machine or a pneumatic hammer, in order to extend the tool's life. The second temperature sensor is located directly on the IMU in the main electronics compartment, and it monitors the electronics temperature. This sensor data will come of use if a malfunction in the electronics occurs, and it is common practice to monitor the temperature of the electronics.

Another issue in the tool electronics design process is autonomy. The tool had to be by specifying a fully autonomous device for the sculptor to express his idea on the stone, uninterrupted, and without the constant use of cables. Therefore, the tool had to integrate devices such as a Bluetooth connectivity module, a micro-SD module for expandable storage capacity, a power supply module with battery recharging ability, and a battery.

Through the development process of the tool, two electronic configurations were designed. The first design was based on an 8-bit microcontroller (Arduino Nano, which has an ATmega328p), a strain gauge in a Wheatstone bridge configuration, a custom signal amplifier unit for the Wheatstone bridge, an IMU (MPU6050), a temperature sensor (DS18S2), a custom 5 volt voltage regulator unit ( $1 \mathrm{m7805)}$, and a micro-SD module.

During the initial test, several problems were identified, such as noise in the amplified signals from the strain gauges, the noise in the gyroscope and accelerometer sensors of the IMU, the lack of battery chargers, and the lack of a wireless communication module (Wi-Fi or Bluetooth). A major issue at this stage was the large sample time due to the low microprocessor capabilities. Another issue was the autonomy of the device, which in this design, the electronics had large power consumption and the built-in battery could not provide. Therefore, an external power supply must be used. The design of the incorporated devices in the smart carving tool is shown in Figure 2.

\subsubsection{Tool's Electronical Improved System Structure}

The new improved design of the tool's electronic devices solved the issues from the initial versions. Priority in this design was given to the optimization of the load measurement unit, the optimization of the firmware sample time, and increasing the tool's autonomy.

To obtain the load measurements as accurately as possible, a strain gauge module capable of measuring and digitizing the signals from the strain gauges in real-time. The strain gauges were configured in a Wheatstone bridge topology. The selected load measurement module DCell (Figure 3, Device No. 2) was an industrial design from Mantracourt, with a minimum dimension factor ideal for integration into the smart carving tool small spaces. DCell uses the Modbus communication protocol to send measurements to other devices. To achieve communication between the DCell and the central process unit, an RS485 communication unit (Figure 3, device No. 4), which allows the operation of the Modbus protocol, was designed, and manufactured. 


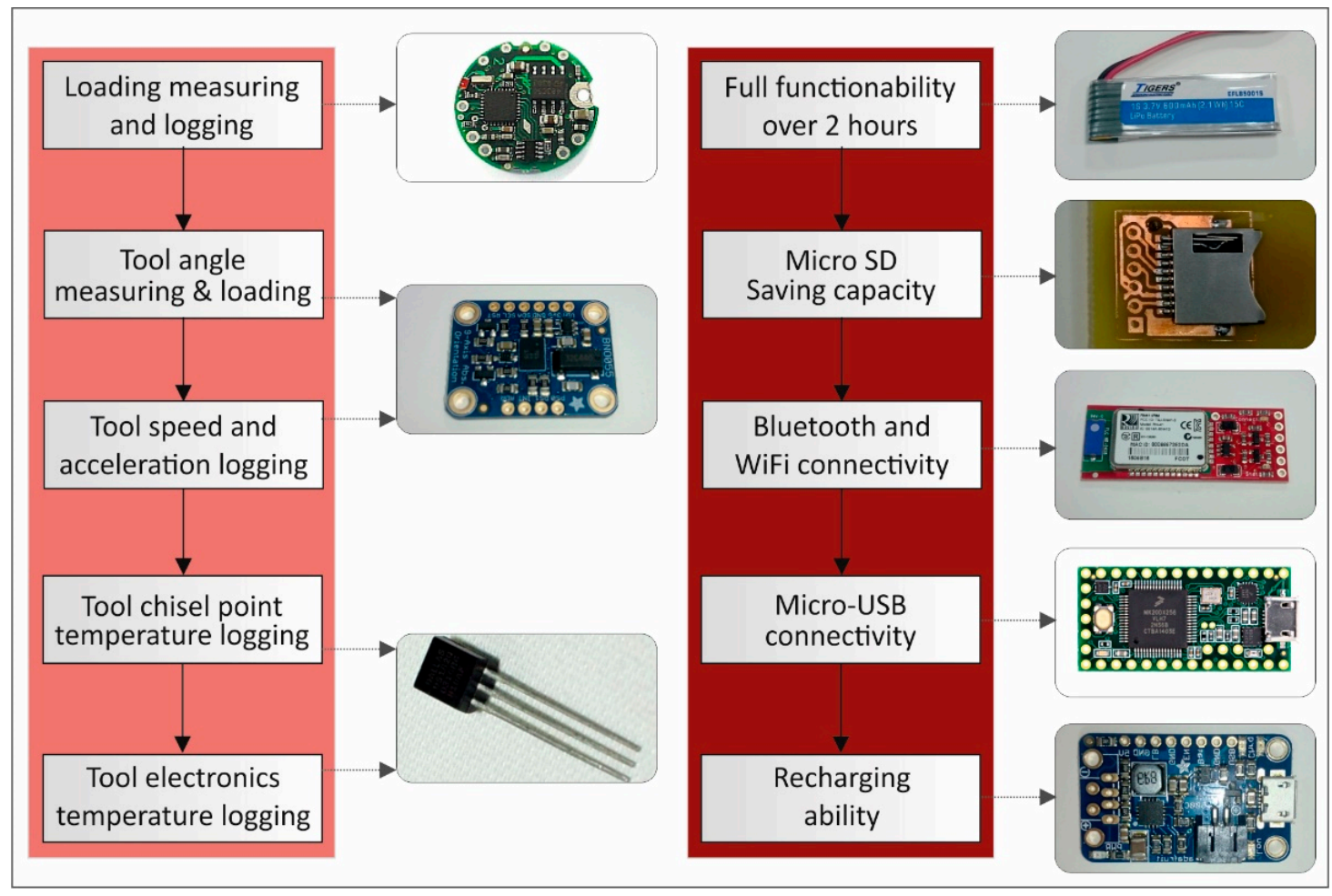

Figure 2. The basic design of the incorporated electronics devices.

In addition, the DCell offered the option for specific temperature sensor interconnection, which could be used both for direct temperature measurement reading and for temperature compensation in the load measurement. Thus, in the tool, compatible with the Dcell temperature sensor DS18S2 (Figure 3, device No. 13) from Maxim Integrated.

A very accurate IMU sensor was selected and integrated into the tool in relation to the linear acceleration and rotation measurement unit. BNO055 (Figure 3, Device No. 3) from Bosch. The module has a three-axis gyroscope sensor, a three-axis accelerometer sensor, and a three-axis magnetometer sensor, with an embedded 32-bit ARM CortexM0 based microprocessor on a single chip. All embedded sensor units are based on microelectromechanical system (MEMS) technology. The embedded microprocessor uses sensor-fusion algorithms to provide accurate data output.

The selected Bluetooth module was BlueSMiRF Gold (Figure 3, device No. 5) by Sparkfun, which can achieve wireless communication between the tool and the host computer for storage and further processing the collected data from the tool sensors. The Bluetooth module uses the unit RN41-3 of Roving Networks Inc. and classifies it as a class 1 Bluetooth unit. However, the module can achieve wireless communication over $100 \mathrm{~m}$ in open air. For the current design, the BlueSMiRF range reached $10 \mathrm{~m}$.

Consequently, all these devices require a fast central processing unit to accomplish all the essential instruction commands and algorithms in a very short time. For the central processing unit, the Teensy 3.2 (Figure 3, device No. 1) development platform was chosen, which was based on an Arm Cortex-M4 family 32-bit architecture microcontroller, clocked at $72 \mathrm{MHz}$. It has all the necessary instructions set and supports all the required communication protocols, such as I2C, SPI, and three different serial communication modules (UART). The I2C protocol is used by the IMU module and SPI by the SD card module. One of the three servers used by the Bluetooth module and the second by the RS485 module for implementation of the Modbus protocol.

Owing to the limited space, a micro-SD card module (Figure 3, Device No. 6) was developed and manufactured. The circuit design of the module supports the SPI communication protocol. 
Energy requirements were met by the central power supply unit, which supplies all individual electronic devices either by a battery or by an external power supply via micro-USB. For the central power supply, PowerBoost500C (Figure 3, device No. 7) from the Ada fruit was integrated. It has a DC-to-DC boost converter, able to increase the output voltage from 3.7 volts to 5.2 volts. In addition, it incorporates a LiPo battery charging circuit, which uses a LiPo type battery (Figure 3, device No. 8) at 3.7 volts and a capacity of $600 \mathrm{mAh}$, with a pick discharge current of up to $15 \mathrm{~A}$. Additionally, the central power supply unit offers the capability for an on/off power switch (Figure 3, device No. 10).

In addition, three additional electronic devices were integrated into the tool. The first device is a simple micro-USB expansion module (Figure 3, device No. 12), which was developed for passing through the serial communication from the central processing unit to the external computer. The micro-USB expansion module is also connected to the central power supply unit to interconnect an external power source. The second device is an RGB-led indicator (Figure 3, device No. 12), which indicates the firmware state of the tool. The third is a push-button (Figure 3, device No. 11) for the mode selection option. Concluding the above, the data that the tool collects, and exports is listed below in Table 1.

Table 1. Tool's electronic sensors data collection table.

\begin{tabular}{cccc}
\hline Number & Parameters & Units & Appears as Graph in Software \\
\hline 1 & Chisel Temperature & ${ }^{\circ} \mathrm{C}$ & Yes \\
2 & Electronics compartment & ${ }^{\circ} \mathrm{C}$ & Yes \\
3 & temperature & Newtons & Yes \\
4 & Chisel Stress Loading & $\mathrm{m} / \mathrm{s}$ & Yes \\
5 & Linear accelerations (all 3 axes) & $\mathrm{m} / \mathrm{s}^{2}$ & Yes \\
6 & Rotational speeds (all 3 axes) & $\mathrm{m} / \mathrm{s}$ & Yes \\
7 & Rotational accelerations (all 3 axes) & $\mathrm{m} / \mathrm{s}^{2}$ & Yes \\
8 & Loading Frequency & $\mathrm{Hz}$ & Yes \\
9 & Power Consumption and status & $\mathrm{mA} / \mathrm{h}$ & Yes \\
\hline
\end{tabular}

\subsection{Smart Carving Software Development}

In addition, an innovative software was developed. The software achieves better and easier control of the tool by the user with a robust interface. The user from the software interface can observe the orientation in space of a three-dimensional representation of the tool in real-time. In addition, all collected data are illustrated real time in individual graphs. Furthermore, it includes features such as start-stop data recording, choosing the connection port, enabling-disabling the representation of the measurement in the individual graphs, internal tool temperature indicator, and IMU calibration status, all in one control panel. The connection between the tool and the software can be achieved either via a physical USB cable or via Bluetooth.

\subsection{Tool's Testing Methodology}

To validate the response of the smart percussion carving tool, its structural behavior, and the accuracy of its electronic devices, a series of compression experiments were conducted initially by benchmarking and validating the part behavior of the strain gauges alongside the electronic devices in multiple compression scenarios, and second, by using the whole tool assembly in combination with sculpting stones.

Compressive tests were performed using an Imada MX2 tensile test machine (Imada Inc., Northbrook, IL, USA) accompanied by an Imada ZTA $2500 \mathrm{~N}$ digital force gauge (Imada Inc., Northbrook, IL, USA) (Figure 4a). The first series of experimental procedures focused on how the tool operates under an externally applied force. Each experiment was designed based on a specific compression scenario. The tool was placed perpendicular to the tension-compression machine without the interchangeable chisel tip, as shown in Figure 4a. In these scenarios, two measurements were acquired and compared: the tool's 
load measurement (measured internally by the tool's load measurement unit) and the force exerted by the machine, captured simultaneously by two external computers. Some of these experimental scenarios are shown in Figure $4 b$,d. In Figure $4 b$, the machine applied loads from 0 to 800 Newtons and back to 0 Newton, with 50 Newtons step. As in the previous measurement, in Figure 4d, the tool was compressed and decompressed with an upper load of 300 Newtons, and the increment was 25 Newtons.

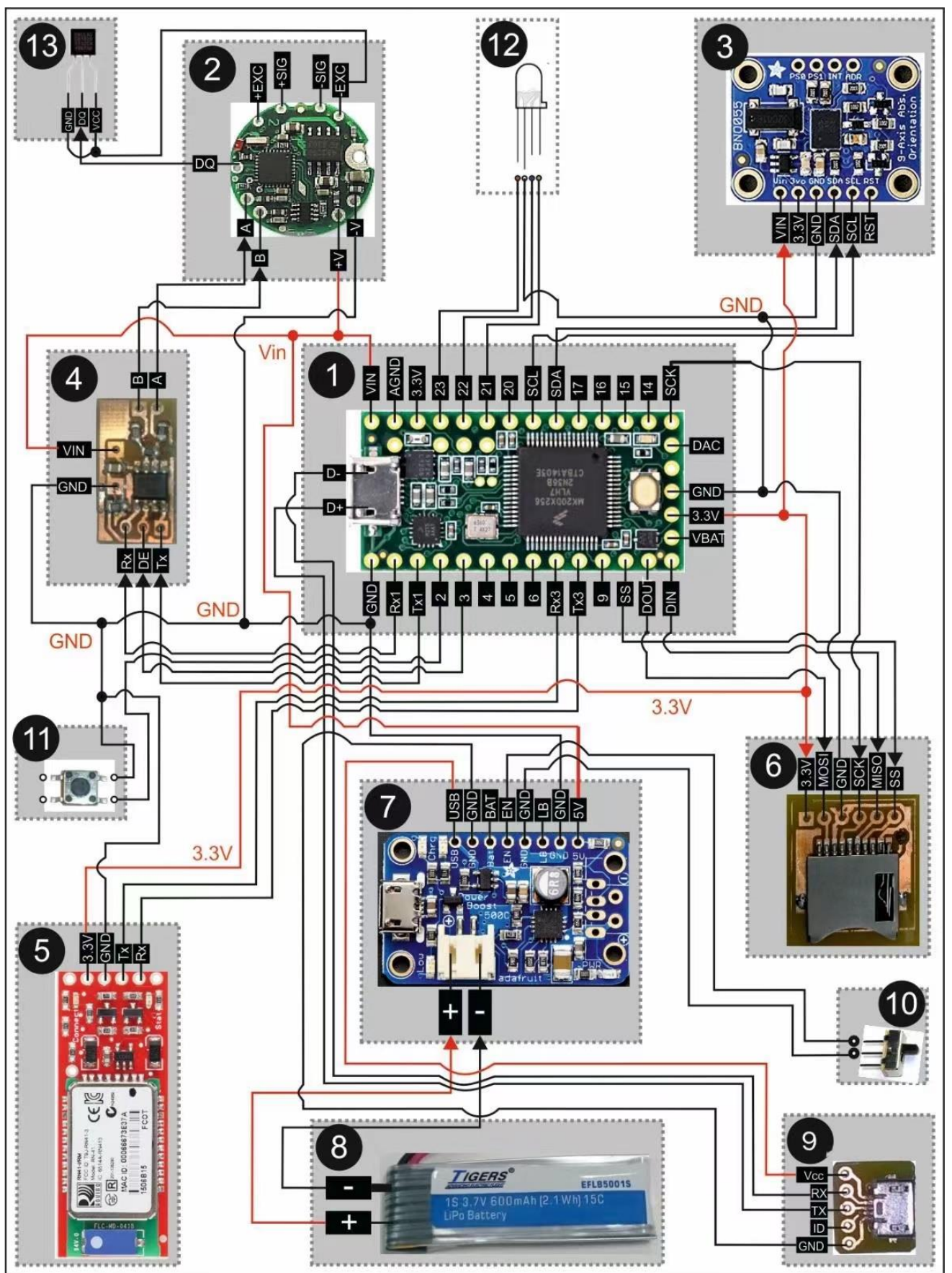

Figure 3. The interconnection diagram of the improved tool's electronic devices, where (1) the central processing unit, (2) the DCell unit, (3) the IMU, (4) the RS485 unit, (5) the Bluetooth module, (6) the micro SD module, (7) the central power supply unit, (8) the LiPo battery, (9) the micro USB expansion module, (10) the power on/off switch, (11) the push button, (12) the red, green and blue light-emitting diode (RGB LED) and (13) the temperature sensor. 

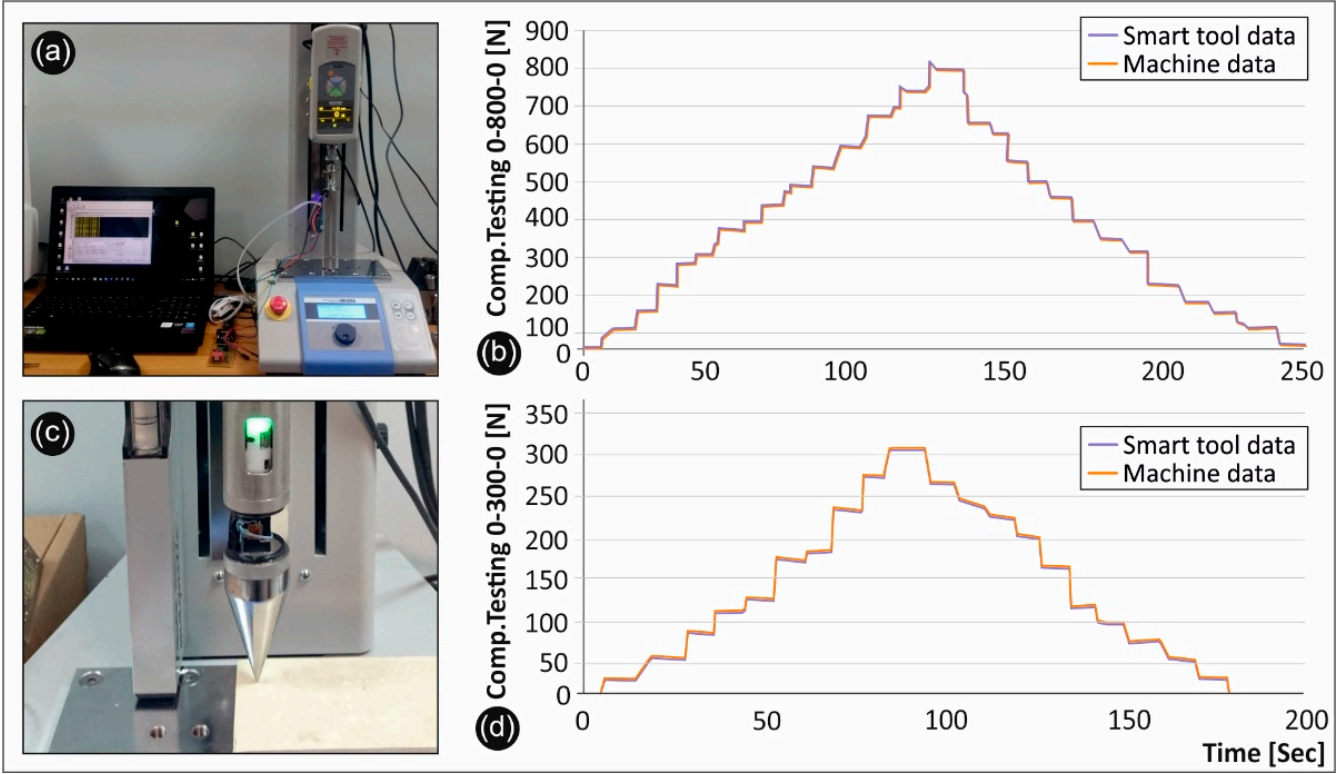

Figure 4. The experimental measurement procedures performed in the tension-compression machine: (a) the first series with the tool's compression scenario, (b) the result graph for scenario 0-800-0 Newtons (c) the second series with the limestone experimental setup and (d) the result for scenario 0-300-0 Newtons.

It must be stated that the smart tool was also tested at the maximum compression forces the tension-compression machine can offer, that is 2500 Newtons. The tools' data are compared to the tension-compression machine data and confirm that the strain gauges are working accurately and are in sync with the tool's loading without failing or without storing any residual forces as a fault, as presented in the Results section of the manuscript.

Regarding calibration, after installing the strain gauges on the smart tool, if there are temperature variations, the tool's material is susceptible to contractions and dilations. So are the over-sensitive strain gauges that are fixed upon the tool. When the tool's status is set to "on" it initially automatically reads the strain gauges' values and sets them to zero (0), compensating the temperatures and materials conditions. It also self-calibrates the gyroscopic sensor. After the auto-calibration (approximately $10 \mathrm{~s}$ after the tool is turned on), the smart tool is ready to accurately measure the aforementioned parameters without the need for further calibration.

The second series of tests focused on two parts. On the one hand the focus was the tool's behavior with natural stones and, on the other hand the focus was how to measure the fracture limit of every one of them. For this scenario, the experimental setup was composed of a natural stone specimen, a tool, a tension-compression machine, and two external computers, to simultaneously capture the two measurements. Thus, the natural stone specimen and tool were placed in the machine, with the interchangeable point chisel initially fitted to the tool. The stone was placed on the base of the machine and the tool was adjusted perpendicular, with the point chisel on the upper surface of the stone (Figure 9c). Therefore, the machine exerts a load on the stone system tool.

Therefore, in the second series, a compression scenario was used in which the initial load was $0 \mathrm{~N}$, the maximum load was $1000 \mathrm{~N}$, and the increment was $100 \mathrm{~N}$. The time required to set in every increment was approximately $10 \mathrm{~s}$. The upper limit of 1000 Newtons was defined after the evaluation that the fracture point of these natural stones was lower. Additionally, the sculptor carves on stones with significantly lower loads from its fracture point to avoid damaging the sculpture. Thus, the load values measured by the tool during the carving process are comparable with this compression scenario.

Five types of natural stone with different mechanical characteristics were selected. The selection was based on natural properties and availability in the Greek marble market. 
These natural stones were limestone of Crete, marble of Naxos, marble of Nestos, marble of Marmara, and granite of Italy. For each stone specimen, a total of six compression measurements were conducted at different points on the surface.

\section{Results}

\subsection{Smart Carving Tool Design and 3D Geometric Models}

Figure 5 depicts the complete assembly model of the smart carving tool, (a) in its first edition, (b) in the second redesigning stage, $(c)$ in the third redesigning stage, and (d) in its final form. The design and manufacturing design were performed on a CAD platform.
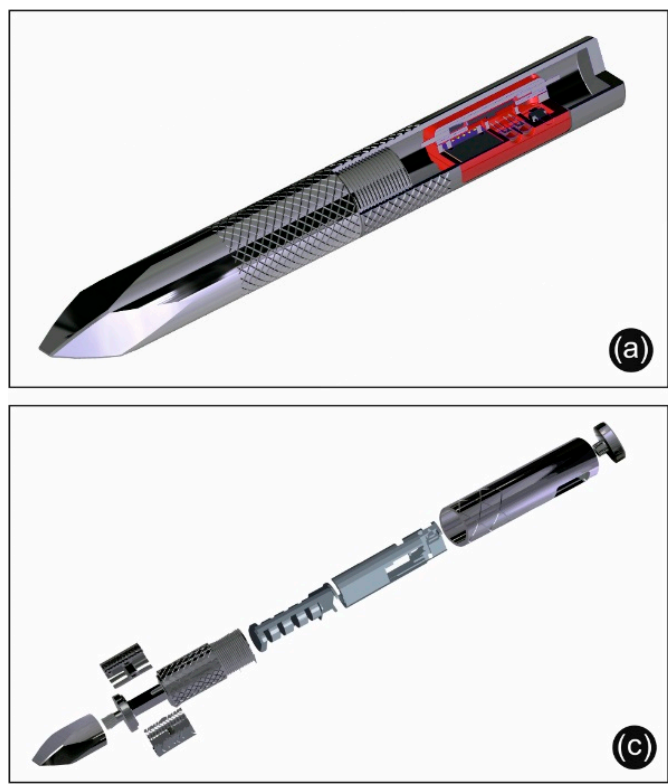

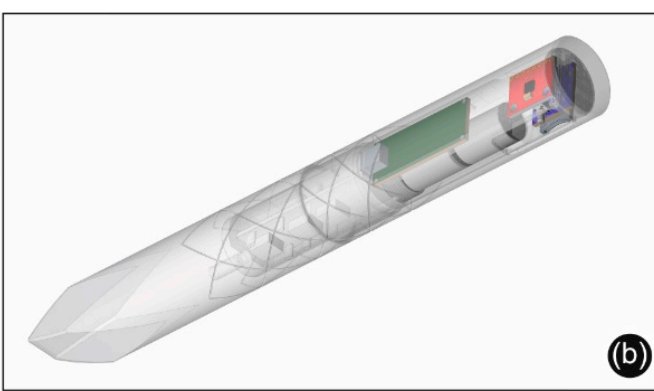

(b)

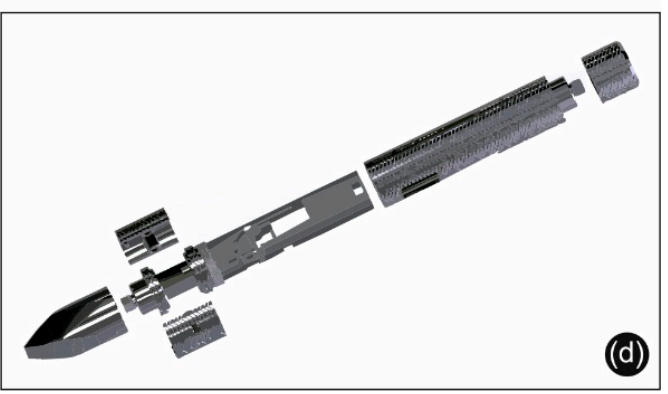

Figure 5. The complete assembly 3D model of the smart carving tool, (a) in its first edition, (b) in the second redesigning stage, $(\mathbf{c})$ in the third redesigning stage and (d) in its final form.

The initial percussion smart carving tool (Figure 5a) was designed in a simple manner to examine the prototype and use it as a base for future, more innovative and more functional models, with little space available for the required electronics. The first 3D model was used to define the minimum and maximum tool body dimensions, with respect to the tool's sizing requirements, the overall chisel length and diameter that were used to define the overall free space for the electronic devices.

Multiple 3D printed prototypes were built and tested. Two more versions of the tool were designed (Figure 5b,c), which gained more space for the electronics inside the tool by redesigning the way the tool's parts were assembled and manufactured. The issue with these versions was again the limited space for the electronic sensors and components and the cable management when it comes to the tool's disassembly for service. The final version of the tool (Figure 5d) provided a smart solution for both manufacturing issues and electronic device placements and disassembly inside the tool. The solution was to manufacture a single polymer part with pockets that would host all the electronics together instead of having multiple parts interconnected via cables.

The final design of this innovative smart tool consists of nine (9) parts, almost all of which can interchange and form a rigid assembly. The complete tool assembly was $250 \mathrm{~mm}$ long with an outer diameter of $30 \mathrm{~mm}$. This smart tool's design embodies the physical requirements set for a fully functional and user-friendly carving tool, including proper dimensioning, effective knurling patterns, mounting counter-bores for easy assembly, and a vast internal usable space of a total $56.42187 \mathrm{~mm}^{3}$ sufficient for assembling the electronics. Figure 6 shows a schematic representation of the smart carving tool use (Figure 6a) and the smart tool parts list (Figure 6b). 

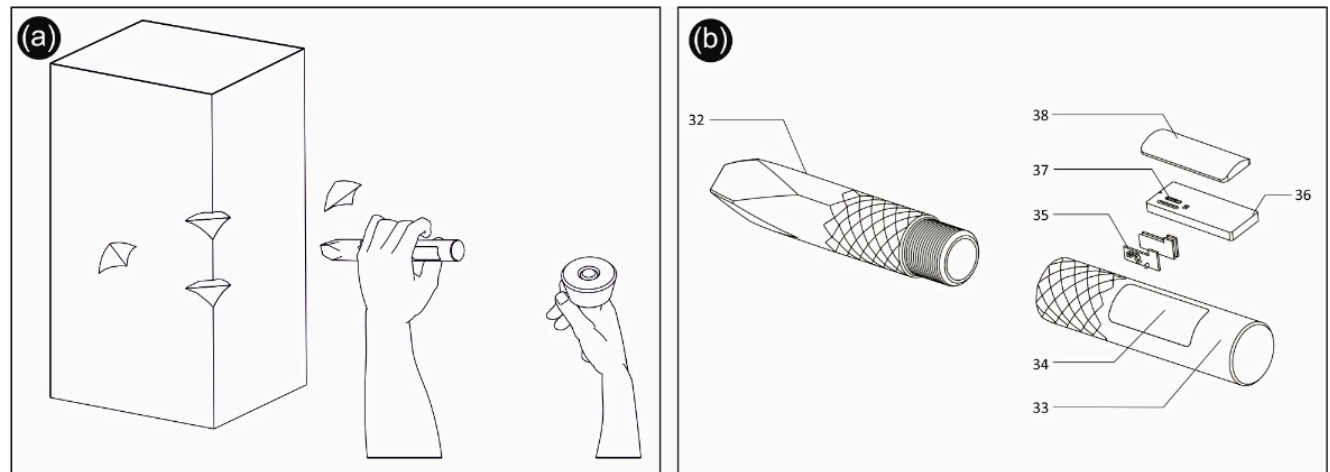

Figure 6. (a) Schematic representation of the smart carving tool use in handheld mode and (b) smart percussion tool's parts list. (Images from the smart percussion tool patent).

\subsection{Smart Carving Tool's Mechanical Parts Manufacturing}

The final parts were manufactured using CNC machinery and are presented in Figure 7. They consist of the main body that was required to host the electronic microdevices (b), the application part of the strain gauges, and its covers, which were used to host the electronic strain gauges (c and d), the percussion disk (f), the flat chisel (e), the tooth chisel (not shown), the point chisel (not shown), and finally the frosting chisel (not shown).
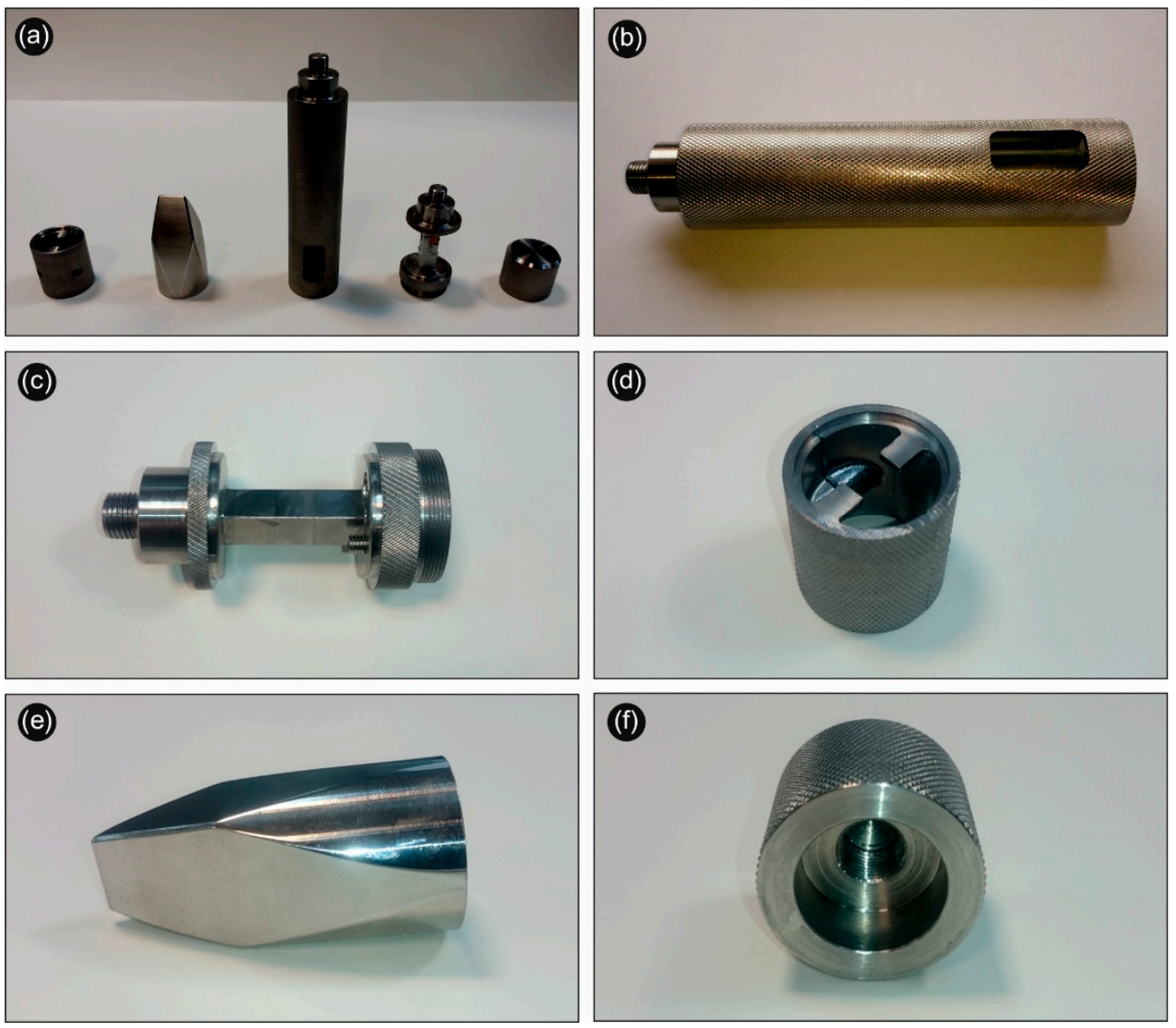

Figure 7. All parts manufactured for the smart percussion carving tool; (a) all parts separated, (b) main electronics host body, (c) strain gauges housing, (d) strain gauges house covers, (e) flat chisel nose part, (f) back chisel disk. 


\subsection{Smart Tool Electrical and Electronic Devices}

The physical tool prototype consists of a total of six sections (Figure 8a), the interchangeable chisel section, the front section, the front section covers, the main part (Figure 8b), the rear impact disc, and the electronic modules retaining section (Figure 7d). This design of the carving tool offers fast and easy assembly/disassembly or component replacement. All the main sections of the tool were manufactured using stainless steel, except for the electronics retaining section, which was manufactured by ertacetal. The front section was designed to install the two half-bridge strain gauges in such an orientation to obtain the axial forces applied to the tool. Likewise, in the front section, a DS18S2 temperature sensor was installed. Furthermore, all the electronic devices are integrated into the electronics retaining section, which in the current tool design attaches to the front section. The main part has an opening to enable the user to interact with the USB expansion module, the SD card module, the power on/off switch, the mode selection button, and the led indicator. In order, the user controls the operation of the tool.
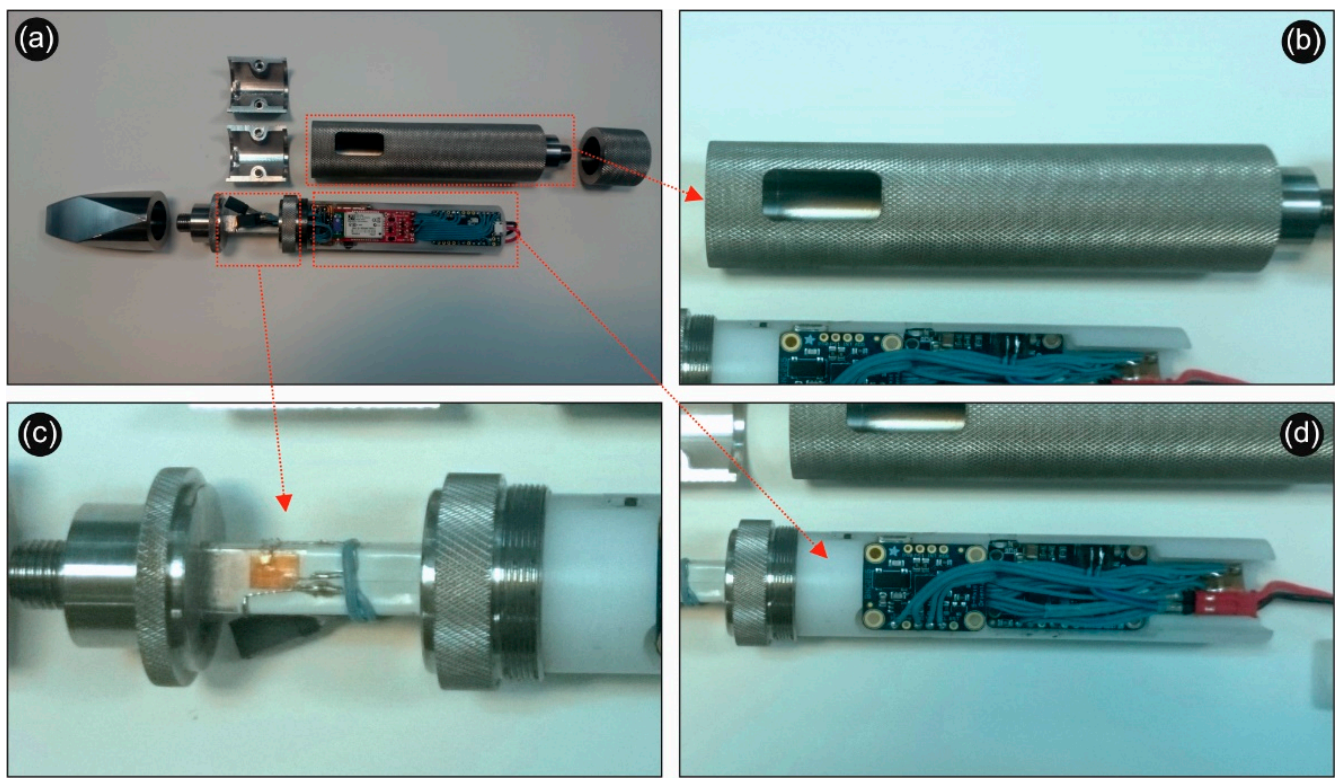

Figure 8. The optimized tool for percussion carving prototype, in fully expanded form (a), the tool's main part (b), the tool's front section illustrating the strain gauge (c), the tool's electronic modules retaining section, $(\mathbf{d})$ the tool's electronics' assembly.

\subsection{Smart Carving Software in Real Use}

The innovative software developed is shown in Figure 9. This software tool provides a graphical interface that shows the 3D geometry and replicates in the computer screen all the tool's movements (speed and acceleration in all axes), and rotations, in all six degrees of freedom. This software tool also shows in real-time all the tool sensors' data and their state.

\subsection{Smart Carving Tool Testing}

Sculpting stones in general, regardless of their type, are inhomogeneous, and their mechanical and physical properties are difficult to define precisely. This is of great importance for the smart carving tool to link the exported variables and effects to each material's properties. Several studies have been conducted to define each stone category's mechanical and physical properties. Studies have shown that the fractural strength of stones depends greatly on grain size [8,9] in parallel with their surface hardening and homogeneity [10].

In both compression test series, the parts of the tool are strictly positioned vertically to the axis of the machine to achieve proper mechanical loading on the axis of the main tool. The results of the first series of compression loading on the strain gauges' part (Figure $4 \mathrm{~b}, \mathrm{~d}$ ) 
show that the tool's force measurements are in complete agreement with the machine's loading profile, and the results are noise-free and of high quality.
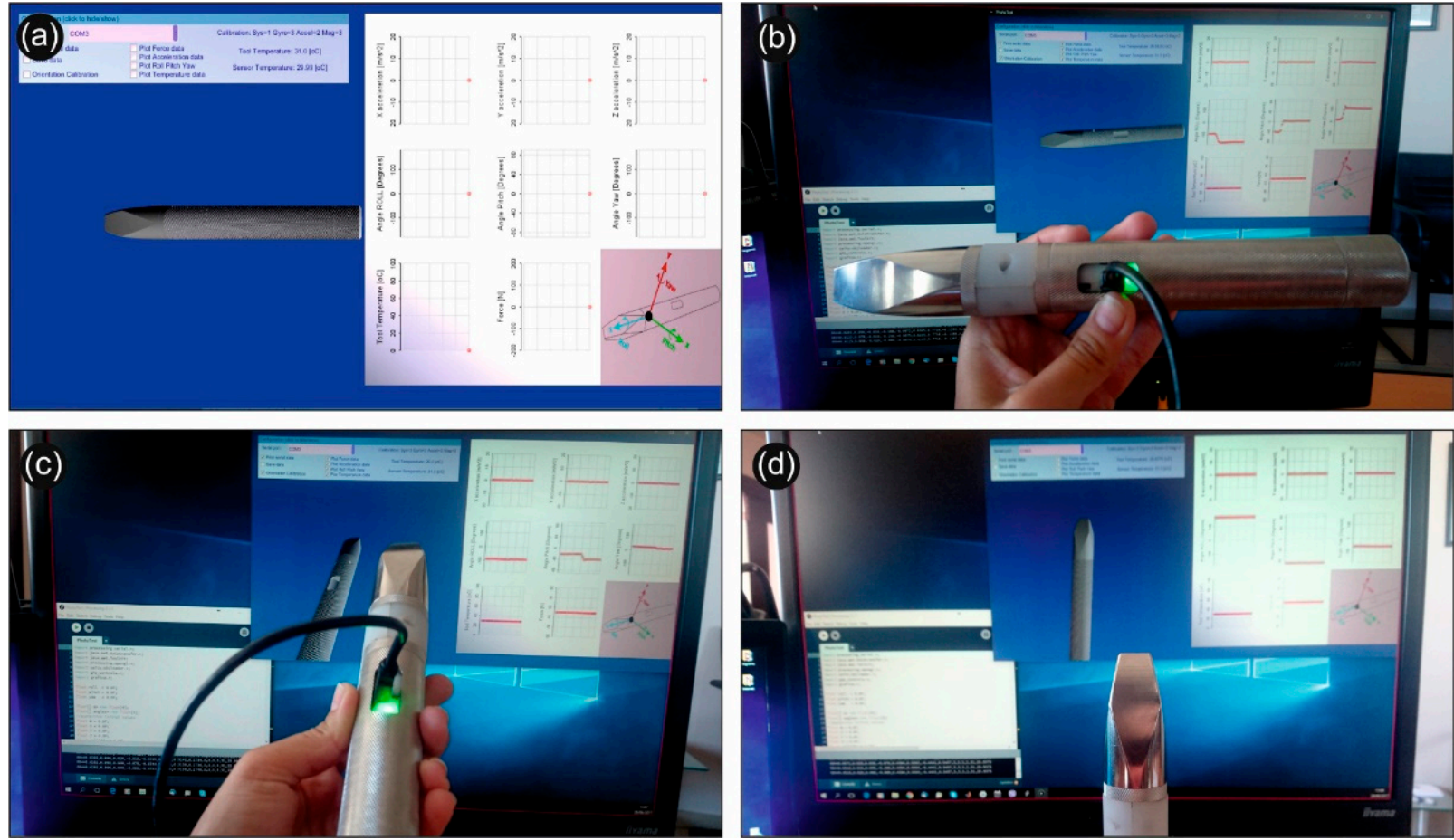

Figure 9. The innovative software developed in this work (a), at the left are located the control panel and the three-dimensional model, at the right are located the individual charts, and at (b-d) are highlights during operation.

In the second series of experiments, the results indicated that the electronic devices within the tool could record the momentary effect of fracture. This was noticeable, because of the momentary relaxation/drop of the machine's heavy loading onto the smart tool that indicates that the chisel point moved inside the material, breaking part of it. After the experiment, there was evidence on the testing stones as well, with parts of them detached. In parallel, the results also indicate that the smart percussion carving tool assembly can withstand high mechanical loading without deformations, while the electronic devices within remain active and functional.

\section{Discussion}

From the overall conduct of experiments and the use of the smart carving tool, it is concluded that the tool can withstand high mechanical loads over multiple charging cycles, retaining its assembly and functionality with no deformations in its structure. In parallel, the electronic devices within have resolution and cycle time to detect and record the momentary phenomenon of impact and fracture regardless of the sculpturing stone, while they remain intact and functional in all stages of percussion carving and high mechanical loading.

The tool's operating and sampling process is stable in all types of experiments and uses, allowing in this manner, stable and proper sampling and recording of all the parameters of the electronic sensor devices and with all recording modes in use. The smart tool designed, manufactured, and tested herein proved to be reliable and entirely compatible with the specifications set in this study. The smart carving tool quantifies the carving process parameters, providing information, which is the first step in modeling and digitizing this artistic process. The caving process art was quantified for the first time in this work. This is the first step to automate the percussion sculpturing process and achieve at the same 
time results in sculptures that can be almost identical to human-made ones. Thus, the improvement is related to the improvement in the sculptures produced via an automated digitally-driven percussion process.

\section{Conclusions}

In this study, a smart percussion carving tool was designed, manufactured, and programmed, capable of mapping the entire carving process, either in the hands of an experienced sculptor or even in a fully automated sculpting machine.

The smart percussion carving tool's key points and its novelty are presented below.

- Its design has been patented as there is no other smart carving tool capable of processing stones via traditional percussion sculpturing.

- The smart tool can measure all the necessary parameters of the percussion carving process, even in the hands of a sculptor, in order to obtain all the critical information that maps this procedure.

- The tool electronic system can measure all the necessary parameters of percussion sculpturing with accuracy and noise.

- The smart percussion carving tool's electronic systems can offer up to three hours of uninterrupted working autonomy, while everything is recorded in the tool's memory card.

- All its parts are precisely manufactured from industrial grade stainless steel, fully interchangeable, leading to long-lasting use even with high and frequent loads.

- The user-friendly software that the smart tool can connect to offers the end-user the ability to handle the tool with more ease and download/erase/analyze the tool's obtained data.

In future work, electric and electronic devices can be further optimized and updated to newer versions. Additionally, more sensors can be added, and the smart carving tool can be further tested in other types of material and tests.

\section{Patents}

Patented under Greek law, with number 1009101 and international number (Int. Cl.): B25D 3/00, B28D 1/26.

Author Contributions: Conceptualization, N.V.; methodology, M.P.; software, A.M. and E.A. validation, N.V. and M.P.; formal analysis, A.M. and E.A.; investigation, A.M.; resources, N.V.; data curation, N.V., A.M. and E.A.; writing-original draft preparation, A.M. and E.A.; writing-review and editing, M.P.; visualization, A.M. and E.A.; supervision, N.V.; project administration, N.V.; funding acquisition, N.V. The manuscript was written through the contributions of all the authors. All authors have read and agreed to the published version of the manuscript.

Funding: This research received no external funding.

Institutional Review Board Statement: Not applicable.

Informed Consent Statement: Not applicable.

Data Availability Statement: The data presented in this study are available upon request from the corresponding author.

Conflicts of Interest: The authors declare no conflict of interest.

\section{References}

1. Rockwell, P. The Art of Stoneworking: A Reference Guide; Cambridge University Press: Cambridge, UK, 1994 ; ISBN 9780521413329.

2. Daas, M.; Wit, A.J. Towards a Robotic Architecture; ORO Editions: San Francisco, CA, USA, 2018; ISBN 9781939621634.

3. Shaked, T.; Bar-Sinai, K.L.; Sprecher, A. Adaptive robotic stone carving: Method, tools, and experiments. Autom. Constr. 2021, 129, 103809. [CrossRef]

4. Gramazio, F.; Kohler, M.; Langenberg, S. Fabricate 2014: Negotiating Design \& Making; UCL Press: London, UK, 2017; ISBN 9781787352148. 
5. Chen, Y.; Han, X.; Okada, M.; Chen, Y. Integrative 3D modelling of complex carving surface. CAD Comput. Aided Des. 2008, 40, 123-132. [CrossRef]

6. Siegesmund, S.; Snethlage, R. (Eds.) Stone in Architecture: Properties, Durability; Springer: Berlin/Heidelberg, Germany, 2014. [CrossRef]

7. Rockwell, P. Stone-carving tools: A stone-carver's view. J. Roman Archaeol. 1990, 3, 351-357. [CrossRef]

8. Brace, W.F. Dependence of fracture strength of rocks on grain size, Fourth Symposium on Rock Mechanics. In Proceedings of the 4th U.S. Symposium on Rock Mechanics (USRMS), University Park, PA, USA, 30 March-1 April 1961; pp. 76, 99-103.

9. William, A. Olsson, Grain Size Dependence of Yield Stress in Marble. J. Geophys. Res. 1974, 79, 4859-4862.

10. Zhang, P.; Li, S.X.; Zhang, Z.F. General relationship between strength and hardness. Mater. Sci. Eng. A 2011, 529, 62-73. [CrossRef] 